\title{
Comparison of radiological scoring systems, clinical scores, neutrophil-lymphocyte ratio and serum C-reactive protein level for severity and mortality in acute pancreatitis
}

\author{
(D) Naciye S Gezer ${ }^{1}$ \\ (iD) Göksel Bengi² \\ (iD) Agah Baran ${ }^{1}$ \\ Dakize E Erkmen ${ }^{2}$ \\ (iD) Ömer S Topalak² \\ (iD) Canan Altay ${ }^{1}$ \\ (iD) Oğuz Dicle
}

\begin{abstract}
1. Department of Radiology, Dokuz Eylül University Faculty of Medicine, İzmir, Turkey 2. Department of Internal Medicine, Dokuz Eylül University Faculty of Medicine, İzmir, Turkey
\end{abstract}

http://dx.doi.org/10.1590/1806-9282.66.6.762

\section{SUMMARY}

Comparison of radiological scoring systems, clinical scores, neutrophil-lymphocyte ratio and serum C-reactive protein level for severity and mortality in acute pancreatitis

BACKGROUND/AIMS: To compare radiological scoring systems, clinical scores, serum C-reactive protein (CRP) levels and the neutrophil-lymphocyte ratio (NLR) for predicting the severity and mortality of acute pancreatitis (AP).

MATERIALS AND METHODS: Demographic, clinical, and radiographic data from 80 patients with AP were retrospectively evaluated. The harmless acute pancreatitis score (HAPS), systemic inflammatory response syndrome (SIRS), bedside index for severity in acute pancreatitis (BISAP), Ranson score, Balthazar score, modified computed tomography severity index (CTSI), extrapancreatic inflammation on computed tomography (EPIC) score and renal rim grade were recorded. The prognostic performance of radiological and clinical scoring systems, NLR at admission, and serum CRP levels at 48 hours were compared for severity and mortality according to the revised Atlanta Criteria. The data were evaluated by calculating the receiver operator characteristic (ROC) curves and area under the ROC (AUROC).

RESULTS: Out of 80 patients, 19 (23.8\%) had severe AP, and 9 (11.3\%) died. The AUROC for the BISAP score was 0.836 (95\%Cl: 0.735 0.937), with the highest value for severity. With a cut-off of BISAP $\geq 2$, sensitivity and specificity were $68.4 \%$ and $78.7 \%$, respectively. The AUROC for NLR was 0.915 (95\% Cl: 0.790-1), with the highest value for mortality. With a cut-off of NLR $>11.91$, sensitivity and specificity were $76.5 \%$ and $94.1 \%$, respectively. Of all the radiological scoring systems, the EPIC score had the highest AUROC, i.e., O.773 (95\% Cl: 0.645-0.900) for severity and 0.851 (95\% Cl: 0.718-0.983) for mortality, with a cut-off value $\geq 6$.

CONCLUSION: The BISAP score and NLR might be preferred as early determinants of severity and mortality in AP. The EPIC score might be suggested from the current radiological scoring systems.

KEYWORDS: Pancreatitis acute necrotizing, Pancreatitis. Mortality, C-reactive protein, Neutrophils. Lymphocyte count.

DATE OF SUBMISSION: 12-Nov-2019

DATE OF ACCEPTANCE: 24-Nov-2019

CORRESPONDING AUTHOR: Göksel Bengi

Department of Gastroenterology, Faculty of Medicine, Dokuz Eylul University Hospital, Mithatpasa Cad. 35340 Inciralti-Izmir, Turkey - Tel.:

+90 5324626972 - Tel.: +90 2324123713

E-mail: drgokselbengi@hotmail.com 


\section{INTRODUCTION}

Acute pancreatitis (AP) is usually a self-limiting disease with minimal systemic effects and good outcomes. However, in $10-20 \%$ of the cases, the clinical course results in systemic inflammatory response syndrome (SIRS) and multiple organ failure ${ }^{1}$. This severe form of the disease is associated with a mortality of up to $50 \%$. Early recognition of patients with the severe presentation of the disease has been shown to improve prognosis and reduce mortality by establishing close monitoring, early intensive therapy, and proper timing of interventions ${ }^{2}$.

Older age, obesity, alcohol and tobacco consumption, hematocrit, serum urea and creatinine levels, a variety of cytokines, chemokines, and other inflammatory response markers have been shown to be associated with severity and mortality of AP. Besides the routine clinical and laboratory data, the presence of systemic inflammatory response syndrome (SIRS), harmless acute pancreatitis score (HAPS), multifactorial clinical and laboratory scoring systems, such as the Ranson score, bedside index of severity in AP (BISAP) score, acute physiology and chronic health evaluation II (APACHE II) score, have been identified to predict severity and prognosis of $\mathrm{AP}^{3-6}$. The Balthazar grade, modified computed tomography severity index (MCTSI), extrapancreatic inflammation on computed tomography (EPIC) score, and renal rim sign are among the radiologic scoring systems used in the assessment of AP prognosis. Among all these current scoring systems, APACHE II (score $\geq 8$ ) is regarded as the gold standard ${ }^{7}$.

Among several serum biochemical markers, serum procalcitonin $(>1.8 \mathrm{ng} / \mathrm{mL})$ and C-reactive protein $(\mathrm{CRP}) \geq 150 \mathrm{mg} / \mathrm{L}$ at 48 hours post-admission have been adopted as prognostic factors for the management of $\mathrm{AP}^{8}$. Also, serum levels of the inflammatory mediators interleukin (IL) 6, 8, and IL-10 have been found to be accurate for predicting persistent organ dysfunction in AP patients ${ }^{9,10}$. However, these serum markers are expensive, not readily available, and cannot adequately predict the prognosis or severity of AP. Recently, neutrophil-lymphocyte ratio (NLR), platelet to lymphocyte ratio, and peripheral blood CD4+ T cell count have been proposed as widely available markers that provide a rapid evaluation of the extent of the inflammatory process in AP patients ${ }^{\mathbf{1 1 , 1 2}}$.

Estimation of the magnitude of the inflammatory response to injury in AP patients during the first 48 hours of hospitalization is valuable to guide the appropriate screening of patients, predict complications, and decide whether or not intensive support will be needed. However, comparative data on the clinical and radiological scoring systems for predicting the outcomes in AP are limited and controversial ${ }^{13}$. The purpose of this study was to compare clinical and radiological scoring systems, the serum CRP level, and NLR in predicting the severity and mortality of AP.

\section{METHODS}

Patient population

The study protocol was approved by the institute ethics committee. The demographic and clinical data of AP patients between the ages of 18-100 years who were admitted to our hospital and hospitalized from 2015 to 2018 were retrospectively collected from our hospital's written and electronic medical records. A total of 80 patients with AP whose computed tomography (CT) images were obtained 48-72 hours after the onset of symptoms and 3-4 weeks after the initial imaging were included in the study.

The patients who presented symptoms for more than 3 days at admission, with incomplete clinical data, early or late initial CT examination were excluded from the study. The patients who did not undergo CT 3-4 weeks after the initial imaging or who had chronic pancreatitis were not included in the study.

\section{Diagnostic criteria and classification of AP}

The criteria for AP diagnosis was the presence of at least two of the following three manifestations: acute onset of characteristic upper abdominal pain (persistent, severe, and usually radiating to the back), elevated levels of pancreatic enzymes (serum amylase and/or lipase > 3 times the upper limit of normal), and findings of ultrasonography (US), CT, or MRI suggesting AP. CT images obtained 3-4 weeks after the initial imaging were assessed to determine the severity of the disease and diagnosis of necrotic pancreatitis.

The etiology of AP was classified as gallstones, alcohol, hyper-triglyceridemia, and others (hypercalcemia, drugs, traumatic, autoimmune, endoscopic procedures). In the remaining cases, the etiology was classified as unknown.

The study group was categorized into mild-moderate and severe AP groups according to the revised Atlanta Criteria ${ }^{14}$. 


\section{Data collection}

HAPS was calculated based on the presence of guarding or rebound tenderness, serum creatinine level (abnormal, $\geq 2 \mathrm{mg} / \mathrm{dL}$ ), and hematocrit level (abnormal, $>43 \%$ in men and $>39.6 \%$ in women) on presentation. The BISAP score was calculated using data from the first 24 hours following admission, based on how many of the following characteristics the patient presented: blood urea nitrogen level $>25$ $\mathrm{mg} / \mathrm{dL}$, impaired mental status, systemic inflammatory response syndrome, age $>60$, and pleural effusion on imaging studies. Each determinant was given one point. The Ranson score was calculated using data from the first 48 hours following admission. The presence of features of systemic inflammatory response syndrome (SIRS) was recorded within 24 hours of admission. NLR at admission and serum CRP level $(\mathrm{mg} / \mathrm{L})$ at 48 hours were also recorded.

CT images obtained 48-72 hours after the onset of symptoms were assessed for radiologic scoring systems. The Balthazar score was determined and MCTSI was calculated by the sum of the Balthazar and necrosis scores. The grades of the Balthazar score were as follows: Grade A (0 points) for normal CT, grade B (1 point) for focal or diffuse pancreatic enlargement, grade $\mathrm{C}$ (2 points) for peripancreatic inflammation or gland abnormalities, grade D (3 points) for single fluid collection, and grade E (4 points) for two or more fluid collections or adjacent gas bubbles. Contrast-enhanced CT images were scored for necrosis as follows: 2 points if regions

TABLE 1. COMPONENTS OF EXTRAPANCREATIC INFLAMMATION ON COMPUTED TOMOGRAPHY SCORE.

\begin{tabular}{l|l} 
Pleural effusion & Points \\
\hline None & 0 \\
\hline Unilateral & 1 \\
\hline Bilateral & 2 \\
\hline $\begin{array}{l}\text { Ascites in any of these locations: perisplenic, perihepatic, interloop, } \\
\text { pelvis }\end{array}$ & 0 \\
\hline None & 1 \\
\hline One location & 2 \\
\hline More than one location & \\
\hline Retroperitoneal inflammation & 0 \\
\hline None & 1 \\
\hline Unilateral & 2 \\
\hline Bilateral & \\
\hline Mesenteric inflammation & 0 \\
\hline Absent & 1 \\
\hline Present &
\end{tabular}

of necrosis were less than the equivalent in size to the pancreatic head $(<30 \%), 4$ points for necrosis of $30-50 \%$ of the gland, and 6 points for necrosis of more than $50 \%$ of the gland. Necrosis was scored as 0 if uniform pancreatic enhancement was observed. The EPIC score was determined based on extrapancreatic complications. The components of the EPIC score are presented in Table 1.

The renal rim grade was scored as grade 1 when increases in the attenuation of the anterior pararenal and the perirenal spaces were observed. Grade 2 was defined as increases in the attenuation of the pancreatic side of the Gerota's fascia (pararenal space). Grade 3 was characterized as increases in the attenuation of both the pararenal and the perirenal spaces.

\section{Statistical analysis}

Statistical analyses were performed using SPSS 20.0 (SPSS Inc, Chicago, Illinois). Continuous variables are presented as mean \pm SD. The prognostic performance of all radiological and clinical scoring systems, NLR, and serum CRP levels were compared for mortality and severity according to the revised Atlanta Criteria. The data were also evaluated regarding the ability of each scoring system to predict AP mortality and severity by calculating receiver operator characteristic (ROC) curves using the MedCalc software (SolidWorks, Concord, MA). The sensitivity, specificity, positive predictive value (PPV), and negative predictive value (NPV) for each individual scoring system were calculated based on the Youden index. The area under the ROC (AUROC) was used to evaluate the discriminative ability of ten parameters (HAPS, BISAP score, Ranson score, NLR, CRP level, Balthazar score, MCTSI, EPIC score, and renal rim grade) for predicting severity and mortality. The association of SIRS with severity and mortality was analyzed using the Chi-square test. A $P$-value $<0.05$ was considered significant.

\section{RESULTS}

The present study enrolled 80 AP patients between the ages of 18 and 85 years (mean $55 \pm 17$ ). Men constituted $42.5 \%$ of the study group. The main cause for AP was cholelithiasis, diagnosed in $57.5 \%$ of patients, followed by alcohol (7.6\%), hyper-triglyceridemia (8.8\%), and others (5.1\%). In the remaining cases (21\%), the etiology was unknown. Necrotic pancreatitis confirmed by CT scan was diagnosed in 26 (32.5\%) patients. Severe AP was diagnosed in 19 (23.8\%) patients, and 9 
of them (11.3\%) died. The patients' mean ages were 52 and 64 years in mild-moderate and severe AP groups, respectively. Nineteen patients (23.8\%) developed persistent organ failure. Nine of them (11.3\%) had pulmonary insufficiency, 4 (5\%) had renal failure, and 6 (7.5\%) developed both pulmonary insufficiency and renal failure.

Comparison of scoring systems, NLR, and CRP levels for severity

The ROC curves of the scoring systems yielded an AUC of 0.616 (95\% CI, 0.474-0.757) for HAPS, 0.836 (95\% CI, 0.735-0.937) for the BISAP score, 0.690 (95\% CI, 0.548-0.831) for the Ranson score, 0.726 (95\% CI, 0.565-0.886) for NLR, 0.728 (95\% CI, 0.578-0.878) for CRP level, 0.651 (95\% CI, 0.521-0.782) for the Balthazar score, 0.619 (95\% CI, 0.495-0.744) for MCTSI, 0.773 (95\% CI, 0.645-0.900) for the EPIC score, and 0.836 (95\% CI, 0.735-0.937) for renal rim grade in predicting severe AP (Figure). The best cutoff values calculated for the HAPS, BISAP score, Ranson score, NLR, CRP level, Balthazar score, MCTSI, EPIC score, and renal rim grade were $>1,1,1,6.66$,
204.3, 3, 3, 5, and 1, respectively. Using these cutoff values, the sensitivity, specificity, PPV, and NPV of various scoring systems in predicting severe AP were calculated. Performance statistics of variables for predicting severity in AP are shown in Table 2. The presence of SIRS was significantly associated with severe AP $(\mathrm{P}=0.002)$.

Comparison of scoring systems, NLR and CRP level for mortality

The receiver-operating characteristic (BOC) curves yielded an AUC of 0.660 (95\% CI, 0.489-0.830) for HAPS, 0.847 (95\% CI, 0.711-0.982) for the BISAP score, 0.669 (95\% CI, 0.479-0.859) for Ranson, 0.915 (95\% CI, 0.790-1) for NLR, 0.799 (95\% CI, 0.558-0.1) for CRP level, 0.690 (95\% CI, 0.548-0.832) for the Balthazar score, 0.615 (95\% CI, 0.481-0.749) for MCTSI, 0.851 (95\% CI, 0.718-0.983) for the EPIC score, and 0.564 (95\% CI, 0.735-0.937) for renal rim grade in predicting severe AP.

The best cutoff values calculated for the HAPS, BISAP score, Ranson score, NLR, CRP level, Balthazar score, MCTSI, EPIC score, and renal rim grade

TABLE 2. PERFORMANCE STATISTICS OF VARIABLES FOR PREDICTING SEVERITY IN ACUTE PANCREATITIS.

\begin{tabular}{l|l|l|l|l} 
Variable & AUC & Cutoff & Sensitivity $^{\star}$ & Specificity $^{\star}$ \\
\hline HAPS & $0.616(0.474-0.757)$ & $\geq 2$ & $57.9(33.5-79.7)$ & $62.3(49-74.4)$ \\
\hline BISAP score & $0.836(0.735-0.937)$ & $\geq 2$ & $68.4(43.4-87.4)$ & $78.7(66.3-88.1)$ \\
\hline Ranson score & $0.690(0.548-0.831)$ & $\geq 2$ & $63.2(38.4-83.7)$ & $68.8(55.7-80.1)$ \\
\hline NLR & $0.726(0.565-0.886)$ & $>6.66$ & $94.4(72.7-99.9)$ & $52.5(39.3-65.4)$ \\
\hline CRP & $0.728(0.578-0.878)$ & $>204.3$ & $75(47.6-92.7)$ & $63.2(49.3-75.6)$ \\
\hline Balthazar score & $0.651(0.521-0.782)$ & $\geq 4$ & $89.5(66.9-98.7)$ & $41(28.6-54.3)$ \\
\hline MCTSI & $0.619(0.495-0.744)$ & $\geq 4$ & $64.7(74-99.9)$ & $41(28.6-54.3)$ \\
\hline EPIC score & $0.773(0.645-0.900)$ & $\geq 6$ & $47.4(24.4-71.1)$ & $82(70-91)$ \\
\hline Renal rim grade & $0.706(0.574-0.839)$ & $\geq 2$ & $85.2(73.8-93)$ \\
\hline
\end{tabular}

${ }^{*} 95 \%$ confidence interval is shown in parentheses. AUC = area under the curve, HAPS = harmless acute pancreatitis score, BISAP = bedside index of severity in acute pancreatitis, $\mathrm{NLR}=$ neutrophil-lymphocyte ratio, $\mathrm{CRP}=$ serum $\mathrm{C}$-reactive protein, $\mathrm{MCTSI}=\mathrm{CT}$ severity index, EPIC = extrapancreatic inflammation on computed tomography

TABLE 3. PERFORMANCE STATISTICS OF VARIABLES FOR PREDICTING MORTALITY IN ACUTE PANCREATITIS.

\begin{tabular}{l|l|l|l|l} 
Variable & AUC & Cutoff & Sensitivity & \\
\hline HAPS & $0.660(0.489-0.830)$ & $\geq 2$ & $66.7(29.9-92.5)$ & Specificity $^{*}$ \\
\hline BISAP score & $0.847(0.711-0.982)$ & $\geq 2$ & $77.8(40-97.2)$ & $73.6(48.3-72)$ \\
\hline Ranson score & $0.669(0.479-0.859)$ & $\geq 3$ & $44.4(13.7-78.8)$ & $83.1(72.3-91)$ \\
\hline NLR & $0.915(0.790-1)$ & $>11.91$ & $61.5(31.6-86.1)$ & $73.8(61.5-84)$ \\
\hline CRP & $0.799(0.558-1)$ & $>229$ & $78.6(49.2-95.3)$ & $70.7(57.3-81.9)$ \\
\hline Balthazar score & $0.615(0.481-0.749)$ & $\geq 4$ & $100(76.8-100)$ & $41.5(29.4-54.4)$ \\
\hline MCTSI & $0.615(0.481-0.749)$ & $\geq 4$ & $100(76.8-100)$ & $40(28-52.9)$ \\
\hline EPIC score & $0.851(0.718-0.983)$ & $\geq 6$ & $78.6(49.2-95.3)$ & $81.5(70-90.1)$ \\
\hline Renal rim grade & $0.564(0.379-0.749)$ & $\geq 2$ & $42.9(17.7-71.1)$ & $81.5(70-90.1)$ \\
\hline
\end{tabular}

${ }^{*} 95 \%$ confidence interval is shown in parentheses. AUC = area under the curve, HAPS = harmless acute pancreatitis score, BISAP = bedside index of severity in acute pancreatitis, $\mathrm{NLR}=$ neutrophil-lymphocyte ratio, CRP = serum C-reactive protein, MCTSI = CT severity index, EPIC = extrapancreatic inflammation on computed tomography 

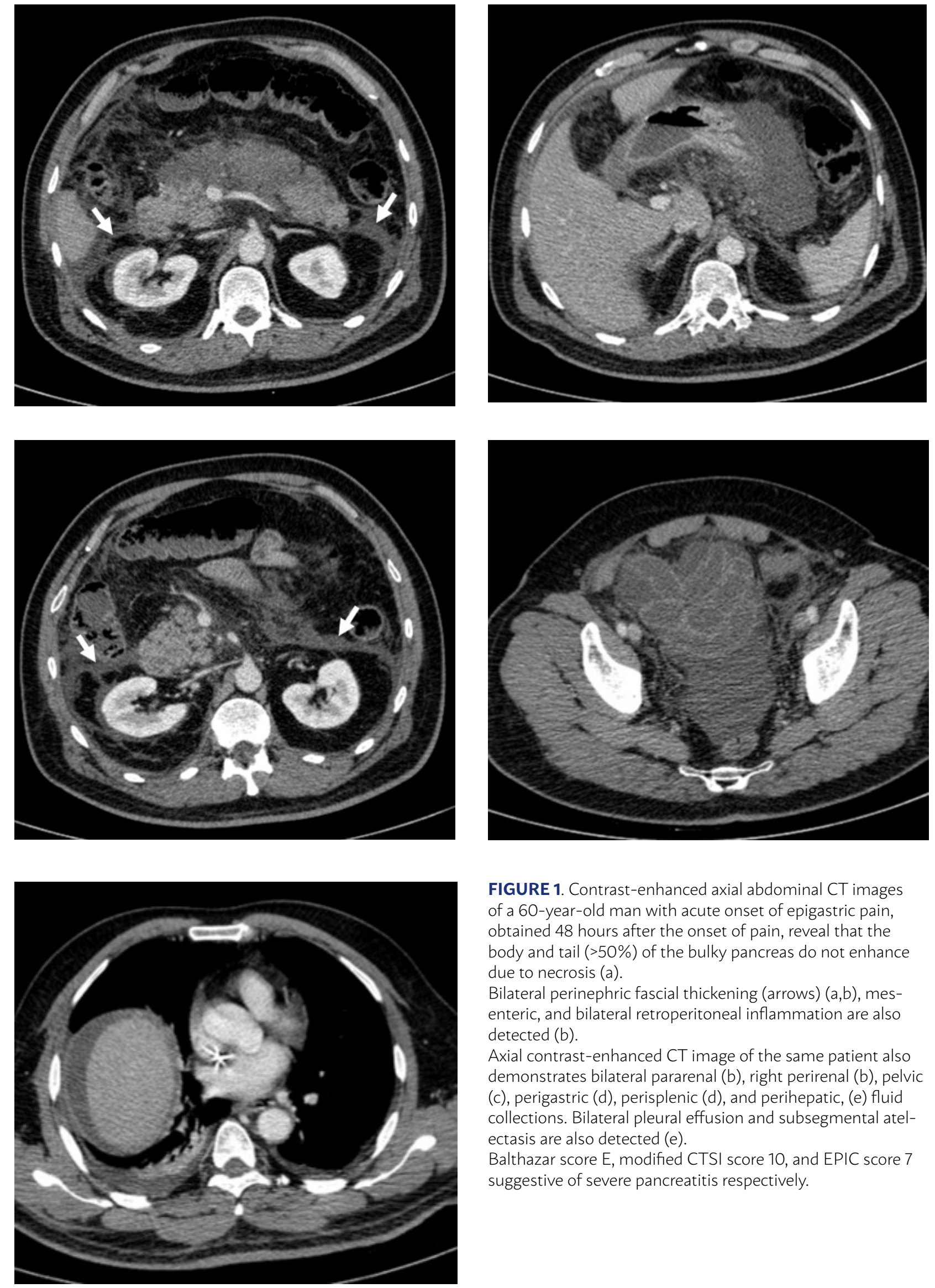

FIGURE 1. Contrast-enhanced axial abdominal CT images of a 60-year-old man with acute onset of epigastric pain, obtained 48 hours after the onset of pain, reveal that the body and tail ( $>50 \%$ ) of the bulky pancreas do not enhance due to necrosis (a).

Bilateral perinephric fascial thickening (arrows) (a,b), mesenteric, and bilateral retroperitoneal inflammation are also detected (b).

Axial contrast-enhanced CT image of the same patient also demonstrates bilateral pararenal (b), right perirenal (b), pelvic (c), perigastric (d), perisplenic (d), and perihepatic, (e) fluid collections. Bilateral pleural effusion and subsegmental atelectasis are also detected (e).

Balthazar score E, modified CTSI score 10, and EPIC score 7 suggestive of severe pancreatitis respectively. 
were $>1,1,2,11.91,229,3,3,5$, and 1 , respectively. Using these cutoff values, the sensitivity, specificity, PPV, and NPV of the various scoring systems in predicting severe AP were calculated. the performance statistics of the variables for predicting mortality in AP are shown in Table 3. The presence of SIRS was significantly associated with mortality $(\mathrm{P}=0.001)$.

\section{DISCUSSION}

In this study, the BISAP score was the most useful among all clinical and radiological scoring systems for severity assessment. A BISAP score $\geq 2$, was the most valuable of all variables to predict severe cases of AP with a sensitivity and specificity of $68.4 \%$ and $78.7 \%$, respectively. On the other hand, NLR had the highest value to predict mortality. With a cutoff value of $>11.91$, the sensitivity and specificity of NLR were $76.5 \%$ and $94.1 \%$, respectively. Among the radiological scoring systems, the EPIC score had the highest value to predict both severity and mortality of AP. With a cut-off value of $\geq 6$ for the EPIC score, the sensitivity and specificity for severity were $63.2 \%$ and $82 \%$, while sensitivity and specificity for mortality were $78.6 \%$ and $81.5 \%$.

In a recent review by Shah et al. ${ }^{7}$, it was declared that the APACHE II scoring system had the highest accuracy for predicting severe AP when compared with other scoring systems. However, the APACHE II scoring system is exhaustive and cannot be widely adopted for AP patients outside the intensive care setting ${ }^{15}$. Also in a previous study, it was demonstrated that the APACHE II score had just a $67 \%$ PPV at $24 \mathrm{~h}$ after admission and was even less accurate for identifying patients with specific complications such as peripancreatic fluid collections or major organ failure $^{16}$. In recent years, researchers have been interested in determining the most practical and accurate parameter indicative of the severity and prognosis of AP. Some researchers have found that no statistically significant pairwise differences were observed between the APACHE-II and the other scoring systems, including CRP value at 24 hours, BISAP, Ranson, and Balthazar scores ${ }^{2,7,17}$. Leung et al. ${ }^{18}$ found that the Ranson and APACHE II scores had lower sensitivity for complications, mortality, and the length of stay for AP than the Balthazar score. On the other hand, in a study of 105 patients with AP, Sharma et al. ${ }^{13}$ found that BISAP (best cutoff value $\geq 3$ ) was more valuable than CT scores (Balthazar score, MCTSI,
EPIC, renal rim) in terms of correlation with organ failure. For this reason, they suggested that performing a CT solely for predicting the severity of AP may not provide additional information to what is already provided by BISAP. In our study, even with a lower cutoff value $(\geq 2)$ we found BISAP was the most useful for severity assessment in comparison with other variables (HAPS, Ranson score, NLR, CRP level, Balthazar score, MCTSI, EPIC score, and renal rim grade). Chen et al. ${ }^{17}$ also found that the best cutoff value for BISAP was 2 in predicting severity. They compared the BISAP, APACHE II, and Ranson scoring systems in 497 patients and found that the BISAP performed similarly to the Ranson and APACHE II scores in predicting severe AP in terms of AUC, sensitivity, and specificity, suggesting that the BISAP is a reliable scoring system for predicting severity (AUC of 0.762 for BISAP, 0.755 for APACHE II, and 0.801 for Ranson).

Chen et al. ${ }^{17}$ also demonstrated that the Balthazar score was superior to other variables (AUC of 0.762 for BISAP, 0.755 for APACHE II, and 0.801 for Ranson) in predicting mortality. On the other hand, Sharma et al. ${ }^{13}$ found that the BISAP had the highest value (AUC 0.90) for predicting mortality in AP compared with the SIRS, Balthazar score, MCTSI, Renal rim grade, and EPIC score. They declared that BISAP 3-5 had 100\% sensitivity and $75.3 \%$ specificity for mortality. In our study, we demonstrated that even a lower BISAP cutoff value $(\geq 2)$ was valuable for predicting mortality (AUC 0.847). On the other hand, Sharma et al. ${ }^{13}$ did include NLR in their study, which was found to be the most valuable for predicting mortality among all variables, including BISAP in our study (AUC 0.915) with a cutoff value $\geq 11.9$.

CRP has also been proven to be a reliable and easily accessible marker in AP, providing good prognostic accuracy for severity assessment, prediction of pancreatic necrosis, and in-hospital mortality when measured at 48-72 hours following hospital admission $^{8,19}$. Thus, $\mathrm{CRP} \geq 15 \mathrm{mg} / \mathrm{dL}$ is adopted as a prognostic factor in AP. However, in our study, another inexpensive and widely available parameter, NLR, had almost the same value as CRP for predicting severe AP and even markedly higher value to predict mortality than CRP value.

Recently, many researchers have investigated the value of NLR in predicting severity and outcomes across a variety of conditions, such as inflammation, cardiovascular disease, neoplastic states, and 
preeclampsia ${ }^{11,20,21}$. As an indicator of uncontrolled SIRS and its progression to multi-organ dysfunction syndrome, increased NLR has also been shown to be associated with poor outcomes in severe AP. Although activation and modulation of neutrophils and platelets play a central role in establishing host defenses in settings of systemic inflammation, excessive inflammatory response results in massive cell transmigration to the pancreas, which in turn results in the destruction of the pancreas and organ failure subsequent to release of aggressive defense molecules $^{22}$. Azab et al. ${ }^{23}$ were the first to demonstrate that NLR was significantly increased in patients who developed severe AP. Jeon and Park ${ }^{24}$ also demonstrated that high baseline NLR was associated with severe AP and organ failure. Han et al. ${ }^{25}$ declared that NLR on admission within $48 \mathrm{~h}$ had the highest AUC for predicting severe AP, with a cut-off value of 6.66 , and NLR was also significantly positively correlated with the Ranson score and hospital stays. Zhang et al. ${ }^{11}$ suggested that high NLR is associated with persistent organ failure, extended duration of intensive care, and also a higher mortality rate. In line with their study, we found that NLR had the highest value to predict mortality compared with other scoring systems and biomarkers. But in contrast with the literature, the BISAP score was the most useful for severity assessment in our study among all the variables. Compatible with our findings, Gulen et al. ${ }^{26}$ found that NLR was not a significant independent prognostic factor for mortality in AP compared with HAPS and red-cell distribution. A superior aspect of our study in comparison with previous literature is that we were able to compare numerous scoring systems and biomarkers.

In line with the literature, the presence of SIRS was found to be significantly associated with AP severity and mortality in this study. Although imaging plays an important role in the diagnosis and management of acute pancreatitis, it does not provide information on systemic inflammatory response and organ failure. In contrast with the literature, the EPIC score accuracy in predicting organ failure was found to be similar to that of BISAP and SIRS scores in a study by Chen et al. ${ }^{27}$; however, it still was not found to be beneficial in differentiating the severity and number of failed organs in the early stage of AP. Since we found that none of the radiologic scoring systems were superior to other clinical, biochemical or hematologic variables, our study also suggests imaging could only be utilized to identify pancreatic or peripancreatic complications and guide therapeutic interventions. Nevertheless, when we made a comparison among radiologic scoring systems including the Balthazar score, MTSI, EPIC, and renal rim grade, an EPIC score $\geq 6$ was the most valuable for predicting both severity and mortality. an EPIC score $\geq 6$ and renal rim grade $\geq 2$ were markedly specific, while a Balthazar score $\geq 4$ and MCTSI $\geq 4$ were markedly sensitive for both severity and mortality. In line with our study, Sharma et al. ${ }^{13}$ also declared that the EPIC score was the most specific (39.2\%) for mortality among all four of these radiologic scoring systems.

Our study had some limitations. First of all, it had a retrospective design. Thus, we were not able to evaluate the parameters which are not routinely used for AP patients in our hospital, such as procalcitonin level. For the same reason, the APACHE II score was not included in the variables of our study. Second, the number of patients enrolled in this study was small because patients with missing data in their hospital files and those without CT imaging at the appropriate time were not included. Another limitation of this study was that the variables were not compared in terms of etiology, even though it could influence the systemic response to AP.

Various scoring systems, biochemical, and hematologic markers are shown to have high predictive accuracy for AP severity and mortality AP in recent studies. In this study, the BISAP with a cut-off value of $\geq 2$ was found to be more valuable than the HAPS, BISAP score, Ranson score, NLR, CRP level, Balthazar score, MCTSI, EPIC score, and renal rim grade for severity assessment, with sensitivity and specificity of $68.4 \%$ and $78.7 \%$, respectively. NLR with a cutoff value of $>11.91$, had the highest value to predict mortality among all variables, with sensitivity and specificity of $76.5 \%$ and $94.1 \%$, respectively. Although radiologic scoring systems do not provide information on systemic inflammatory response and organ failure, the EPIC score had the highest value to predict both severity and mortality of AP in comparison with the others. The EPIC score, with a cut-off of $\geq$ 6 , presented sensitivity and specificity of $63.2 \%$ and $82 \%$, respectively, for severity, and $78.6 \%$ and $81.5 \%$, respectively for severity. In conclusion, the BISAP score and NLR might be preferred as early determinants of severity and mortality in AP. The EPIC score might be suggested among current radiological scoring systems. 


\section{Ethics Committee Approval}

This study was approved by the Ethics Committee of the Dokuz Eylül University School of Medicine.

\section{Author Contributions}

Concept - N.S.G., G.B., Ö.S.T., O.D.; Design - N.S.G., G.B., Ö.S.T.; Supervision - Ö.S.T., O.D.; Materials G.B., Ö.S.T., P.E.E.; Data Collection and/or Processing - A.B., P.E.E.; Analysis and/or Interpretation - N.S. G.
A.B., P.E.E.; Literature Review - A.B., P.E.E.; Writing N.S.G., G.B.; Critical Review - Ö.S.T., O.D.

\section{Conflict of Interest}

The authors have no conflicts of interest to declare.

\section{Financial Disclosure}

The authors declare that this study has received no financial support.

\section{RESUMO}

Comparação dos sistemas de escores radiológicos, escores clínicos razão neutrófilo/linfócito e níveis séricos de proteína C-reativa para determinação da gravidade e mortalidade em casos de pancreatite aguda

OBJETIVO: Comparar sistemas de escores radiológicos, escores clínicos, os níveis séricos de proteína C-reativa (PCR) e a razão neutrófilo/ linfócitos (RNL) como métodos de previsão de gravidade e mortalidade em casos de pancreatite aguda (PA).

MATERIAIS E MÉTODOS: Dados demográficos, clínicos e radiográficos de 80 pacientes com PA foram avaliados retrospectivamente. Os valores de Harmless Acute Pancreatitis Score (HAPS), Síndrome da Resposta Inflamatória Sistêmica (SIRS), Índice de Gravidade na Pancreatite Aguda à Beira do Leito (BISAP), escore de Ranson, escore de Balthazar, Índice Modificado de Gravidade por Tomografia Computadorizada (CTSI), escore de Inflamação Extrapancreática em Tomografia Computadorizada (EPIC) e grau renal foram registrados. O desempenho prognóstico dos sistemas de escores clínicos e radiológicos e RNL no momento da internação e os níveis séricos de PCR após 48 horas foram comparados quanto à gravidade, de acordo com os critérios de Atlanta revisados e mortalidade. Os dados foram avaliados pelo cálculo das curvas ROC e da área sob a curva ROC (AUROC).

RESULTADOS: De 80 pacientes, 19 (23,8\%) tinham PA grave e 9 (11,3\%) morreram. A AUROC para o escore BISAP foi de 0,836 (95\% CI: 0.735-0.937), com o valor mais alto de gravidade. Com um valor de corte de BISAP $\geq 2$, a sensibilidade e a especificidade foram de $68,4 \%$ e 78,7\%, respectivamente. A AUROC para o a RNL foi de 0,915 (95\% Cl: 0.790-1), com o valor mais alto de mortalidade. Com um valor de corte de RNL > 11,91, a sensibilidade e a especificidade foram de 76,5\% e 94,1\%, respectivamente. Entre os sistemas de escore radiológico, o EPIC apresentou o maior valor de AUROC, 0,773 (95\% Cl: 0.645-0.900) para gravidade e 0,851 (95\% Cl: 0.718-0.983) para mortalidade com um valor de corte $\geq 6$.

CONCLUSÃO: O escore BISAP e a RNL podem ser preferíveis como determinantes precoces de gravidade e mortalidade na PA. O escore EPIC pode ser sugerido entre os atuais sistemas de escores radiológicos.

PalaVRas-ChaVe: Pancreatite necrosante aguda, Pancreatite, Mortalidade, Proteína C-reativa, Neutrófilos, Contagem de linfócitos.

\section{REFERENCES}

1. Ferreira AF, Bartelega IA, Urbano HC, Souza IK. Acute pancreatitis gravity predictive factors: which and when to use them? Arq Bras Cir Dig. 2015;28(3):207-11

2. Cho JH, Kim TN, Chung HH, Kim KH. Comparison of scoring systems in predicting the severity of acute pancreatitis. World J Gastroenterol. 2015;21(8):2387-94.

3. Papachristou GI, Clermont G, Sharma A, Yadav D, Whitcomb DC. Risk and markers of severe acute pancreatitis. Gastroenterol Clin North Am. 2007;36(2):277-96.

4. Wilson C, Heath DI, Imrie CW. Prediction of outcome in acute pancreatitis: a comparative study of APACHE II, clinical assessment and multiple factor scoring systems. Br J Surg. 1990;77(11):1260-4.

5. Wu BU, Johannes RS, Sun X, Tabak Y, Conwell DL, Banks PA. The early prediction of mortality in acute pancreatitis: a large population-based study. Gut. 2008;57(12):1698-703.

6. Cho SK, Jung S, Lee KJ, Kim JW. Neutrophil to lymphocyte ratio and platelet to lymphocyte ratio can predict the severity of gallstone pancreatitis. BMC Gastroenterol. 2018;18(1):18.
7. Shah AP, Mourad MM, Bramhall SR. Acute pancreatitis: current perspectives on diagnosis and management. J Inflamm Res. 2018;11:77-85.

8. Cardoso FS, Ricardo LB, Oliveira AM, Canena IM, Horta DV, Papolia AL, et al. C-reactive protein prognostic accuracy in acute pancreatitis: timing of measurement and cutoff points. Eur J Gastroenterol Hepatol. 2013;25(7):784-9.

9. Brand M, Götz A, Zeman F, Behrens G, Leitzmann M, Brünnler T, et al. Acute necrotizing pancreatitis: laboratory, clinical, and imaging findings as predictors of patient outcome. AJR Am J Roentgenol. 2014;202(6):1215-31.

10. Jiang S, Ni M, Zhang Y, Wu Y, Lu X. Association of IL-10 polymorphisms with acute pancreatitis. Int J Clin Exp Med. 2016;9(12):23702-6.

11. Zhang Y, Wu W, Dong L, Yang C, Fan P, Wu H. Neutrophil to lymphocyte ratio predicts persistent organ failure and in-hospital mortality in an Asian Chinese population of acute pancreatitis. Medicine. 2016;95(37):4746.

12. Yang Z, Zhang Y, Dong L, Yang C, Gou S, Yin T, et al. The reduction of peripheral blood CD4+ T cell indicates persistent organ failure in acute pancreatitis. PLoS One. 2015;10(5):e0125529.

13. Sharma V, Rana SS, Sharma RK, Kang M, Gupta R, Bhasin DK. A study of radiological scoring system evaluating extrapancreatic inflammation with 
conventional radiological and clinical scores in predicting outcomes in acute pancreatitis. Ann Gastroenterol. 2015;28(3):399-404.

14. Thoeni RF. The revised Atlanta classification of acute pancreatitis: its importance for the radiologist and its effect on treatment. Radiology. 2012;262(3):751-64.

15. Dambrauskas Z, Gulbinas A, Pundzius |, Barauskas G. Value of the different prognostic systems and biological markers for predicting severity and progression of acute pancreatitis. Scand J Gastroenterol. 2010;45(7-8):959-70

16. Larvin M, McMahon MJ. APACHE-II score for assessment and monitoring of acute pancreatitis. Lancet. 1989;2(8656):201-5.

17. Chen L, Lu G, Zhou Q, Zhan Q. Evaluation of the BISAP score in predicting severity and prognoses of acute pancreatitis in Chinese patients. Int Surg. 2013;98(1):6-12.

18. Leung TK, Lee CM, Lin SY, Chen HC, Wangh HJ, Shen LK, et al. Balthazar computed tomography severity index is superior to Ranson criteria and APACHE II scoring system in predicting acute pancreatitis outcome. World | Gastroenterol. 2005;11(38):6049-52.

19. Staubli SM, Oertli D, Nebiker CA. Laboratory markers predicting severity of acute pancreatitis. Crit Rev Clin Lab Sci. 2015;52(6):273-83.

20. Gemenetzis G, Bagante F, Griffin JF, Rezaee N, Javed AA, Manos LL, et al. Neutrophil-to-lymphocyte ratio is a predictive marker for invasive malignancy in intraductal papillary mucinous neoplasms of the pancreas. Ann Surg. 2017;266(2):339-45
21. Gezer C, Ekin A, Ertas IE, Ozeren M, Solmaz U, Mat E, et al. High first-trimester neutrophil-to-lymphocyte and platelet-to-lymphocyte ratios are indicators for early diagnosis of preeclampsia. Ginekol Pol. 2016;87(6):431-5.

22. Bermejo-Martin JF, Tamayo E, Ruiz G, Andaluz-Ojeda D, Herrán-Monge R, Muriel-Bombin A, et al; EXPRESS (Expresión Génica en Sepsis) and GRECIA (Grupo de Estudios y Análisis en Cuidados Intensivos) groups. Circulating neutrophil counts and mortality in septic shock. Crit Care. 2014;18(1):407.

23. Azab B, Jaglall N, Atallah JP, Lamet A, Raja-Surya V, Farah B, et al. Neutrophil-lymphocyte ratio as a predictor of adverse outcomes of acute pancreatitis. Pancreatology. 2011;11(4):445-52.

24. Jeon TJ, Park JY. Clinical significance of the neutrophil-lymphocyte ratio as an early predictive marker for adverse outcomes in patients with acute pancreatitis. World J Gastroenterol. 2017;23(21):3883-9.

25. Han C, Zeng J, Lin R, Liu J, Qian W, Ding Z, et al. The utility of neutrophi to lymphocyte ratio and fluid sequestration as an early predictor of severe acute pancreatitis. Sci Rep. 2017;7(1):10704.

26. Gulen B, Sonmez E, Yaylaci S, Serinken M, Eken C, Dur A, et al. Effect of harmless acute pancreatitis score, red cell distribution width and neutrophil/ lymphocyte ratio on the mortality of patients with nontraumatic acute pancreatitis at the emergency department. World J Emerg Med. 2015;6(1):29-33.

27. Chen C, Huang Z, Li H, Song B, Yuan F. Evaluation of extrapancreatic inflammation on abdominal computed tomography as an early predictor of organ failure in acute pancreatitis as defined by the revised Atlanta classification. Medicine (Baltimore). 2017;96(15):e6517. 